\title{
REFORMULATION OF TRANSMISSION AND REFLECTION PROBLEMS IN TERMS OF QUANTUM WAVE IMPEDANCE FUNCTION
}

\author{
O. I. Hryhorchak (D) \\ Professor Ivan Vakarchuk Department for Theoretical Physics, \\ Ivan Franko National University of Lviv, \\ 12, Drahomanov St., Lviv, UA-79005, Ukraine \\ e-mail: Orest.Hryhorchak@lnu.edu.ua \\ (Received 17 September 2021; in final form 08 November 2021; accepted 07 December 2021; \\ published online 29 December 2021)
}

\begin{abstract}
On the basis of 1D Schrödinger equation, a non-linear first-order differential equation (Riccati type) for a quantum wave impedance function was derived. The advantages of this approach were discussed and demonstrated for the case of a single rectangular barrier. Both the scattering and the bound states problems were reformulated in terms of quantum wave impedance. The application of the method for solving these problems was considered. The expressions for the reflection and transmission coefficients were found on the basis of the quantum wave impedance approach.
\end{abstract}

Key words: Schrödinger equation, quantum mechanical wave impedance, scattering, tunneling

DOI: https://doi.org/10.30970/jps.25.4001

\section{INTRODUCTION}

The term "impedance" was introduced by Oliver Heaviside [1] in 1886. A few years later, Arthur Kennelly represented it with complex numbers [2]. Usually, we understand impedance as some extension of a resistance concept which is applicable in DC circuits, to the AC electrical circuits. It has a real part and an imaginary part. The imaginary part is caused by both the effects of the induction of voltages in conductors by magnetic fields and the electrostatic storage of charge induced by voltages between conductors. At the same time, resistance forms the real part of the impedance.

It would be a mistake to state that impedance is only related to electrical circuits. Impedance is a generalized concept which is widely used in different areas of science from classical mechanics, electricity and acoustics to geology and medicine. Generally, a wave impedance characterizes the resistance of the media to the propagation of wave processes in it. For example, mechanical impedance relates velocities with forces which act on a mechanical system and indicates how much a mechanical system resists the motion caused by a harmonic force [3]. Acoustic impedance is a measure of the resistance which a medium has to the acoustic flow. This resistance is a reaction to the acoustic pressure applied to the medium [4].

Wave impedance of an electromagnetic wave is the resistance which an electromagnetic wave experiences when it propagates along a transmission line or through a medium including vacuum. Wave impedance can be defined through the ratio between transverse components of the electric and magnetic fields [5].

In all abovementioned cases, the equations which describe the propagation of wave processes in different media have the same form and it gives us the reason to introduce the impedance concept in all these cases.

So now a question arises: what about the wave function and the Schrödinger equation which describes its behavior? Can we talk about the applicability of the wave impedance concept in this case? The answer is definitely yes, because the Schrödinger equation has the same form as equations which describe wave processes in mechanics, acoustics, electrical circuits etc. It also means that we can take the results obtained for a wave impedance in other areas of physics and apply them to quantum mechanical systems. Khondker, Khan and Anwar were the first who did it [6]. They used an analogy with an electrical transmission line to introduce the concept of quantum wave impedance. Further papers demonstrated the efficacy of the quantum wave impedance approach for an analysis of quantum-mechanical structures with a potential which has a complicated spatial structure.

Although this approach is quite good and allows applying the relations obtained within the transmission line theory to quantum mechanical systems, it is still worth getting the relations for a quantum mechanical impedance from the first principles because this will open other dimensions of the application of the quantum wave impedance method. But neither in paper [6] nor in the further papers (see, for example, [7-23]) dedicated to the quantum wave impedance, a systematic introduction of this concept or a consistent theory based on the first principles was proposed. So, the aim of this article is to fill this gap by constructing such a theory starting from the Schrödinger equation; to demonstrate an application of quantum wave impedance for solving both the scattering and the bound states problems.

\section{A NON-LINEAR DIFFERENTIAL EQUATION FOR THE QUANTUM WAVE IMPEDANCE}

We start from the Schrödinger equation and rewrite it in the following way

$$
\left(\frac{\psi^{\prime}(x)}{\psi(x)}\right)^{\prime}+\left(\frac{\psi^{\prime}(x)}{\psi(x)}\right)^{2}=\frac{2 m(U(x)-E)}{\hbar^{2}} .
$$




\section{O. I. HRYHORCHAK}

Multiplying both sides of the previous equation by $\hbar /(\mathrm{im})$ and introducing the following notation

$$
Z(x)=\frac{\hbar}{i m}\left(\frac{\psi^{\prime}(x)}{\psi(x)}\right)
$$

we get a first order differential equation for the quantum wave impedance

$$
\frac{d Z(x)}{d x}+i \frac{m}{\hbar} Z^{2}(x)=i \frac{2}{\hbar}(E-U(x)) .
$$

Note that this equation is a nonlinear Riccati equation. It seems that Hartree [24] was the first who used a logarithmic derivative of the wave function and introduced a non-linear differential equation for it on the basis of the Schrödinger equation. He applied this concept to study an atom with a non-Coulomb central field.

In formula (3) $Z(x)$ is called the quantum wave impedance. A multiplier $\hbar /(\mathrm{im})$ is introduced so that the expression for the probability current density has the simplest form:

$$
\begin{aligned}
j & =\frac{\hbar}{2 i m}\left(\psi^{*} \frac{d \psi}{d x}-\psi \frac{d \psi^{*}}{d x}\right)=\frac{1}{2}|\psi(x)|^{2}\left(Z(x)+Z^{*}(x)\right) \\
& =|\psi(x)|^{2} \operatorname{Re}[Z(x)] .
\end{aligned}
$$

Using (2), we get the relation between a quantum wave impedance and a wave function in the following form

$$
\psi(x)=C \exp \left[\frac{i m}{\hbar} \int^{x} Z\left(x^{\prime}\right) d x^{\prime}\right],
$$

where $C$ is the constant. Thus, we can express the probability current density only through a quantum wave impedance function $Z(x)$ :

$$
\begin{aligned}
j & =\left|C \exp \left[\frac{i m}{\hbar} \int^{x} Z\left(x^{\prime}\right) d x^{\prime}\right]\right|^{2} \operatorname{Re}[Z(x)] \\
& =|C|^{2} \exp \left[-\frac{2 m}{\hbar} \int^{x} \operatorname{Im}\left[Z\left(x^{\prime}\right)\right] d x^{\prime}\right] \operatorname{Re}[Z(x)]
\end{aligned}
$$

Naturally, the following question arises. What are the advantages of a non-linear first order equation (Riccati type equation for a quantum wave impedance) over a linear second order equation (Schrödinger equation)? The main advantage is that the solution of a firstorder differential equation contains only one undefined constant while a second order equation has two undefined constants. So the procedure of the consecutive calculation of quantum wave impedance is much easier compared to a wave function calculation because instead of two matching conditions it is enough to use only one.

If we define the value for a quantum wave impedance function $Z(x)$ at an arbitrary point $x=x_{0}$ (we call it a boundary condition), then its values at other points are defined by the equation (3) and the corresponding matching condition. Moreover we might introduce two functions, $Z^{+}\left(x \mid x_{0}\right)$ and $Z^{-}\left(x \mid x_{0}\right)$, namely

$$
Z(x)=\left\{\begin{array}{l}
Z^{-}\left(x \mid x_{0}\right), x<x_{0} \\
Z^{+}\left(x \mid x_{0}\right), x>x_{0}
\end{array}\right.
$$

so both of them belong to the solutions of the equation (3) and aren't necessarily equal. It is also not necessary to use the same value of $x_{0}$ for both $Z^{-}$and $Z^{+}$functions. Often it is appropriate to consider the following functions

$$
Z^{-}(x \mid b), x<b, \quad Z^{+}(x \mid a), x>a,
$$

where $b>a$. Their role we will be discussed later.

\section{SCATTERING CASE}

Dealing with the propagation of a quantum particle, we can distinguish between two cases, namely a scattering case and a bound states one. In this section, we will show how to use the obtained equation (3) for solving a scattering task.

We say that we have a scattering case if such $x_{0}$ exists that the wave incidenting on the left has the value of energy bigger than the potential energy at any point of the area $\left(-\infty, x_{0}\right)$. In the case of a wave incidenting on the right, the condition of the scattering case is very similar: $\exists x_{0}, \forall x \geq x_{0}, U(x)<E$. Note that these conditions don't take into account infinite periodic systems, which have to be considered separately.

A solution of the equation (3) in a scattering case exists for any value of $E$ and relates the probability current density of the incidenting wave to the reflected and transmitted ones. A situation of particular interest is the case of resonant tunneling when the reflection probability current density is equal to zero.

On the basis of formula (6), we see that for having a constant value of probability current density $j$ in the whole region, the value of

$$
\ln (\operatorname{Re}[Z(x)])-\frac{2 m}{\hbar} \int^{x} \operatorname{Im}\left[Z\left(x^{\prime}\right)\right] d x^{\prime}=\text { const }
$$

has to be constant. This expression relates the real part of the quantum wave impedance function to the imaginary one. If const $=0$, we have a full reflection in a studied system or a bound state. At the same time, the following relation

$$
\ln (\operatorname{Re}[Z(x)])-\frac{2 m}{\hbar} \int^{x} \operatorname{Im}\left[Z\left(x^{\prime}\right)\right] d x^{\prime}=\ln \left(z_{0}\right)
$$

is a condition for finding resonant levels, where $z_{0}$ is the characteristic impedance of the region $x<x_{0}, x_{0} \rightarrow$ $-\infty$, when a wave incidents on the left. Note that $z_{0}$ is real and it is reasonable to rewrite the previous condition in the following form

$$
\ln \left(\frac{\operatorname{Re}[Z(x)]}{z_{0}}\right)=\frac{2 m}{\hbar} \int^{x} \operatorname{Im}\left[Z\left(x^{\prime}\right)\right] d x^{\prime} .
$$

Potentials which satisfy this condition (11) for an arbitrary energy value (within the scattering case) are called reflectionless potentials [25]. For an arbitrary potential, we can find only discrete energy values which satisfy this condition (11). But it is not convenient to calculate the energy values using (11). So it would be good to find an 
easier way of finding resonant levels. For this let's consider the following potential

$$
U(x)=\left\{\begin{array}{cc}
U_{1}, & x \leq a \\
f(x), & a<x<b \\
U_{2}, & x \geq b
\end{array}\right.
$$

and two quantum wave impedance functions $Z^{-}(x \mid b)$ and $Z^{+}(x \mid a)$ [see (8)]. Then in the case of resonant levels, for arbitrary $x_{0} \in[a, b]$ we get:

$$
\begin{aligned}
& \ln \left(\frac{\operatorname{Re}\left[Z^{+}\left(x_{0} \mid a\right)\right]}{z_{0}}\right)=\frac{2 m}{\hbar} \int^{x_{0}} \operatorname{Im}\left[Z^{+}\left(x^{\prime} \mid a\right)\right] d x^{\prime} \\
& \ln \left(\frac{\operatorname{Re}\left[Z^{-}\left(x_{0} \mid b\right)\right]}{z_{0}}\right)=\frac{2 m}{\hbar} \int^{x_{0}} \operatorname{Im}\left[Z^{-}\left(x^{\prime} \mid b\right)\right] d x^{\prime}
\end{aligned}
$$

Subtracting one equation from the other we obtain that

$$
\begin{aligned}
& \ln \left(\frac{\operatorname{Re}\left[Z^{+}\left(x_{0} \mid a\right)\right]}{\operatorname{Re}\left[Z^{-}\left(x_{0} \mid b\right)\right]}\right) \\
& =\frac{2 m}{\hbar} \int^{x_{0}} \operatorname{Im}\left[Z^{+}\left(x^{\prime} \mid a\right)-Z^{-}\left(x^{\prime} \mid b\right)\right] d x^{\prime} .
\end{aligned}
$$

Now taking into account that this equation should be valid for arbitrary $x_{0} \in[a, b]$ and that in the case of a wave incidenting on the left, the following equation holds $Z^{+}(a \mid a)=z_{0}$, we get

$$
\begin{aligned}
& \operatorname{Re}\left[Z^{+}\left(x_{0} \mid a\right)\right]=\operatorname{Re}\left[Z^{-}\left(x_{0} \mid b\right)\right], \\
& \operatorname{Im}\left[Z^{+}\left(x_{0} \mid a\right)\right]=\operatorname{Im}\left[Z^{-}\left(x_{0} \mid b\right)\right]
\end{aligned}
$$

or finally

$$
Z^{-}\left(x_{0} \mid b\right)=Z^{+}\left(x_{0} \mid a\right), \quad x_{0} \in[a, b] .
$$

Although we derived this condition for a potential of form (12), it is still valid for a scattering problem with an arbitrary potential.

It is worth saying that the resonant tunneling of waves is important in the formation of the characteristics of wave structures, especially a spectral selection, which allows providing extremely high signal decoupling in operating and non-operating areas of frequency and energy.

\section{BOUND STATES CASE}

If such $x_{1}$ and $x_{2}$ exist that the energy of the wave is less than the potential energy in each point of the region: $x \in\left(-\infty, x_{1}\right) \cup\left(x_{2}, \infty\right)$, then we have a problem which is qualitatively different from the scattering one. It is a bound states case, but note that this condition does not include infinite periodic systems, which have to be considered separately. A solution of equation (3) exists for any value of energy $E$, but only for discrete values of $E$ the solution for the quantum wave impedance function $Z(x)$ corresponds to the wave function $\psi(x)$ which can be normalized per unit. To normalize a wave function, it is necessary at least that $\psi(x) \sim x^{\alpha}$ with $\alpha<-1$, when $x \rightarrow \pm \infty$. It means that $i m Z(x) x / \hbar$ must be less than -1 , when $x \rightarrow \pm \infty$ :

$$
\frac{i m}{\hbar} Z(x) x<-1, \quad x \rightarrow \pm \infty .
$$

The condition for finding eigenvalues of energy is the same we got for the scattering case (16). We will demonstrate this using the potential energy of form (12). Assume that we have solutions of the Schrödinger equation

$$
\begin{aligned}
& -\frac{\hbar^{2}}{2 m} \frac{d^{2} \psi(x)}{d x^{2}}+U(x) \psi(x)=E \psi(x), \\
& E<\min \left(U_{1}, U_{2}\right)
\end{aligned}
$$

in the region $[a, b]$ both to the right and to the left of the arbitrary point $x_{0} \in[a, b]$ and $\Psi_{+}\left(x_{0} \mid x\right)$ is the solution to the left of the point $x_{0}, \Psi_{-}\left(x_{0} \mid x\right)$ is the solution to the right of the same point. Both solutions can be depicted as a linear combination of two linearly independent functions:

$$
\begin{aligned}
& \Psi_{+}\left(x_{0} \mid x\right)=A_{+} \psi(x)+B_{+} \phi(x), \\
& \Psi_{-}\left(x_{0} \mid x\right)=A_{-} \psi(x)+B_{-} \phi(x) .
\end{aligned}
$$

When $x \leq a$, we have the solution of the Schrödinger equation in the form

$$
\Psi_{\mathrm{L}}(x)=C_{\mathrm{L}} \exp \left[\varkappa_{1}(x-a)\right],
$$

and for $x \geq b$

$$
\Psi_{\mathrm{R}}(x)=C_{\mathrm{R}} \exp \left[-\varkappa_{2}(x-b)\right],
$$

where $\varkappa_{1}=\sqrt{2 m\left(U_{1}-E\right)} / \hbar, \varkappa_{2}=\sqrt{2 m\left(U_{2}-E\right)} / \hbar$.

By applying boundary conditions at points $a$ and $b$ one defines $A_{+}, B_{+}, A_{-}, B_{-}$through $C_{\mathrm{R}}$ and $C_{\mathrm{L}}$, because one has to satisfy both a wave function and its first derivative matching at each interface. So

$$
A_{+} \psi(a)+B_{+} \phi(a)=C_{\mathrm{L}}, \quad A_{+} \psi^{\prime}(a)+B_{+} \phi^{\prime}(a)=\varkappa_{1} C_{\mathrm{L}}
$$

and

$$
A_{-} \psi(b)+B_{-} \phi(b)=C_{\mathrm{R}}, \quad A_{-} \psi^{\prime}(b)+B_{-} \phi^{\prime}(b)=-\varkappa_{2} C_{\mathrm{R}} .
$$




\section{O. I. HRYHORCHAK}

Thus

$$
\begin{aligned}
& \Psi_{+}\left(x_{0} \mid a\right)=C_{\mathrm{L}}\left(\frac{\phi^{\prime}(a)-\phi(a) \varkappa_{1}}{\psi(a) \phi^{\prime}(a)-\psi^{\prime}(a) \phi(a)} \psi\left(x_{0}\right)+\frac{\psi^{\prime}(a)-\psi(a) \varkappa_{1}}{\psi^{\prime}(a) \phi(a)-\psi(a) \psi^{\prime}(a)} \phi\left(x_{0}\right)\right), \\
& \Psi_{-}\left(x_{0} \mid b\right)=C_{\mathrm{R}}\left(\frac{\phi^{\prime}(b)+\phi(b) \varkappa_{2}}{\psi(b) \phi^{\prime}(b)-\psi^{\prime}(b) \phi(b)} \psi\left(x_{0}\right)+\frac{\psi^{\prime}(b)+\psi(b) \varkappa_{2}}{\psi^{\prime}(b) \phi(b)-\psi(b) \psi^{\prime}(b)} \phi\left(x_{0}\right)\right) .
\end{aligned}
$$

Now at point $x_{0} \in[a, b]$ one has to satisfy the following matching conditions

$$
\Psi_{+}\left(x_{0} \mid a\right)=\Psi_{-}\left(x_{0} \mid b\right), \quad \Psi_{+}^{\prime}\left(x_{0} \mid a\right)=\Psi_{-}^{\prime}\left(x_{0} \mid b\right) .
$$

Note that these conditions are valid only if the potential at $x_{0}$ point is not singular. In the case of a zero-range singular (at $x_{0}$ point) potential, we would have other matching conditions, which depend on the type of singularity.

The solution for $C_{\mathrm{L}}$ and $C_{\mathrm{R}}$ exists only if

$$
\frac{\Psi_{+}\left(x_{0} \mid a\right)}{\Psi_{+}^{\prime}\left(x_{0} \mid a\right)}=\frac{\Psi_{-}\left(x_{0} \mid b\right)}{\Psi_{-}^{\prime}\left(x_{0} \mid b\right)} .
$$

It is a condition for finding eigenvalues of energy. Multiplying both sides by $\hbar /(\mathrm{im})$ one gets:

$$
Z^{+}\left(x_{0} \mid a\right)=Z^{-}\left(x_{0} \mid b\right),
$$

where $x_{0}$ is an arbitrary point from the region $(a, b)$.

It isn't an unexpected result, since a standing wave is a superposition of two waves of equal amplitudes propagating in opposite directions. This fact explains the equality of impedances $Z^{-}$and $Z^{+}$at an arbitrary point $x_{0} \in(a, b)$.

\section{SOLUTION FOR A CONSTANT VALUE OF POTENTIAL ENERGY}

In this section we are going to find a solution of equation (3) in the region with constant potential energy. Assume that the potential energy is equal to $U_{0}$ and the total energy of the particle is equal to $E$. This is one of the simplest cases but at the same time it is very important and it has many applications especially in the area of numerical calculations.

If $U(x)=U_{0}$, we can separate variables in equation (3) and get that

$$
\frac{i \hbar}{m} \frac{d Z}{Z^{2}-z_{0}^{2}}=d x
$$

where $z_{0}=\sqrt{2\left(E-U_{0}\right) / m}$ is the characteristic impedance of the region with constant potential energy $U_{0}$. After the integration of both parts of the previous relation

$$
\frac{\hbar}{i m z_{0}} \operatorname{artanh}\left(\frac{Z(x)}{z_{0}}\right)=x+x_{0},
$$

it is easy to get a solution for a quantum wave impedance function

$$
Z(x)=z_{0} \tanh \left(\gamma_{0} x+\phi\right),
$$

where $\gamma_{0}=i m z_{0} / \hbar$ is the magnitude which characterizes the wave propagating in the medium with the characteristic impedance $z_{0}, \phi$ is the constant phase which depends on the boundary conditions for a quantum wave impedance function. Generally, $\gamma_{0}$ is a complex quantity, and we also use both $k_{0}\left(i k_{0}=\gamma_{0}\right)$ when $\gamma_{0}$ is fully imaginary and $\varkappa_{0}\left(\varkappa_{0}=\gamma_{0}\right)$ in the case when $\gamma_{0}$ is fully real.

Let us consider the behaviour of $Z(x)$ when $x \rightarrow \pm \infty$ in two cases: $\gamma_{0}$ is real and $\gamma_{0}$ is imaginary. If $\gamma_{0}$ is real, then

$$
\lim _{x \rightarrow \pm \infty} Z(x)=\lim _{x \rightarrow \pm \infty} z_{0} \tanh \left(\gamma_{0} x+\phi\right)= \pm z_{0} .
$$

In this case, we have the attenuation of a wave function when $x \rightarrow \pm \infty$ and the condition which we obtained in the previous section

$$
\lim _{x \rightarrow \pm \infty} \frac{i m}{\hbar} Z(x) x<-1
$$

is satisfied when $i z_{0}<0$ ( $z_{0}$ is fully imaginary). It also means that for the potential of form (12) we must choose the boundary conditions for $Z(x), x \in[a, b]$, in the following form:

$$
Z(a)=-z_{1}, \quad Z(b)=z_{2},
$$

where $z_{1}=\sqrt{2\left(E-U_{1}\right) / m}>0$ and $z_{2}=$ $\sqrt{2\left(E-U_{2}\right) / m}>0$ are the characteristic impedances of the regions $x<a$ and $x>b$ consequently.

Indeed, a wave function $\psi(x)=C \exp \left[\frac{i m}{\hbar} \int^{x} Z\left(x^{\prime}\right) d x^{\prime}\right]$ can be normalized only if we choose $Z(a)=-z_{1}$ and $Z(b)=z_{2}$ because it gives $\psi(x) \rightarrow 0$, when $x \rightarrow \pm \infty$.

If $\gamma_{0}$ is imaginary, then after an appropriate representation of $\tanh (x)$ function it becomes obvious that solution (30) contains a linear combination of plane waves which propagate in opposite directions:

$$
\begin{aligned}
Z(x) & =z_{0} \frac{\exp \left[i k_{0} x+\phi\right]-\exp \left[-i k_{0} x-\phi\right]}{\exp \left[i k_{0} x+\phi\right]+\exp \left[-i k_{0} x-\phi\right]} \\
& =z_{0} \frac{\exp \left[i k_{0} x\right]-r \exp \left[-i k_{0} x\right]}{\exp \left[i k_{0} x\right]+r \exp \left[-i k_{0} x\right]},
\end{aligned}
$$

where $i k_{0}=\gamma_{0}$ ( $k_{0}$ is real), $r=\exp (-2 \phi)$ is a wave amplitude reflection coefficient.

For the potential energy of form (12), when the wave incidents on the left, there is no reason for $Z(x)$ to be dependent on $x$, when $x \geq b$. It is possible if we put $\phi= \pm \infty$. But only $\phi=\infty$ is acceptable because only 
then we have the wave moving in the positive direction of $x$ axis.

$$
\psi(x)=\exp \left[\frac{i m}{\hbar} \int^{x} z_{0} d x^{\prime}\right]=C \exp \left[i k_{2} x\right], x \geq b
$$

where $i k_{2}=\gamma_{2}$ ( $k_{2}$ is real). Indeed, as we remember $r=\exp [-2 \phi]$ in the case of a wave which incidents on the left. If $\phi=\infty$ then $r=0$. It means that a wave is moving without reflection, which is correct for the region $x>b$. As a result we get the right-side boundary condition for a quantum wave impedance function: $Z(b)=z_{2}>0$.

By the same reasoning in the case of a wave incidenting on the right, we have to choose $\phi=-\infty$. Thus, the leftside boundary condition is $Z(a)=-z_{1}<0$. Indeed, in the case of $\phi=-\infty$ we have $r=\infty$, which means that there is no wave moving in the positive direction of $x$, so a wave is moving to the left without any reflection, which is correct for the region $x<a$.

\section{TRANSMISSION AND REFLECTION COEFFICIENTS}

The quantitative expressions of wave scattering are two magnitudes, namely, a wave amplitude reflection coefficient $r$ and a wave amplitude transition coefficient $t$. The wave amplitude reflection coefficient describes how much of an incidenting wave is reflected. It is equal to the ratio of the amplitude of the reflected wave to the amplitude of the incidenting wave. The wave transmi- ssion amplitude coefficient relates the amplitude of a transmitted wave to the amplitude of the incidenting wave.

Having $r$ and $t$, we can calculate both reflection $R=$ $|r|^{2}$ and transmission $T=|t|^{2}$ probabilities. The other definition of these magnitudes is as follows:

$$
T=\frac{\left|j_{+}\right|}{|j|}, \quad R=\frac{\left|j_{-}\right|}{|j|}
$$

where $j$ is the probability density current of an incidenting wave, $j_{+}$is the probability density current of a transmitted wave and $j_{-}$is the probability density current of a reflected wave.

Let's consider a very simple example, namely, a potential step, and find $r, t, R, T$ for this case. So we have a potential energy in the following form

$$
U(x)=\left\{\begin{array}{cc}
U_{1}, & x \leq x_{0} \\
U_{2}>U_{1}, & x>x_{0}
\end{array}\right.
$$

and assume that the wave incidenting on the left has the energy $U_{1}<E<U_{2}$. There are two regions of the constant potential and each of them has its characteristic impedance ( $z_{1}$ and $z_{2}, z_{1}$ is real and $z_{2}$ is imaginary) and a wave number $\left(k_{1}\right.$ and $\varkappa_{2}, k_{1}$ and $\varkappa_{2}$ are real). In these two regions, we calculate the probability density current on the basis of formula (4) and taking into account that $\phi=\phi_{\mathrm{R}}+i \phi_{I}$. We get

$$
Z_{1}(x)=z_{1} \tanh \left(i k_{1} x+\phi_{1}\right)=\frac{z_{1}}{2} \frac{\sinh \left(2 \phi_{\mathrm{R}}\right)}{\sinh ^{2}\left(\phi_{\mathrm{R}}\right)+\cos ^{2}\left(k_{1} x+\phi_{I}\right)}+i \frac{z_{1}}{2} \frac{\sin \left(2\left(k_{1} x+\phi_{I}\right)\right)}{\sinh ^{2}\left(\phi_{\mathrm{R}}\right)+\cos ^{2}\left(k_{1} x+\phi_{I}\right)}
$$

and

$$
\int^{x} \operatorname{Im}\left[Z_{1}\left(x^{\prime}\right)\right] d x^{\prime}=-\frac{z_{1}}{k_{1}} \ln \left[\sinh ^{2}\left(\phi_{\mathrm{R}}\right)+\cos ^{2}\left(k_{1} x+\phi_{I}\right)\right] .
$$

So, finally

$$
|j|-\left|j_{-}\right|=z_{1} \frac{|C|^{2}}{2} \sinh \left(2 \phi_{\mathrm{R}}\right)=z_{1}|\tilde{C}|\left(1-\exp \left(-4 \phi_{\mathrm{R}}\right)\right)
$$

where $|\tilde{C}|^{2}=\frac{1}{2}|C|^{2} \exp \left(2 \phi_{\mathrm{R}}\right)$.

We find the value of $\phi_{\mathrm{R}}$ from the matching condition at the $x_{0}$ point:

$$
z_{1} \tanh \left(i k_{1} x_{0}+\phi\right)=z_{2}
$$

Taking into account that

$$
\begin{aligned}
\tanh \left(i k_{1} x_{0}+\phi\right) & =\frac{1-\exp \left[-2\left(i k_{1} x_{0}+\phi\right)\right]}{1+\exp \left[-2\left(i k_{1} x_{0}+\phi\right)\right]} \\
& =\frac{1-r \exp \left[-2 i k_{1} x_{0}\right]}{1+r \exp \left[-2 i k_{1} x_{0}\right]}
\end{aligned}
$$

we get

$$
r=\exp (-2 \phi)=\exp \left[2 i k_{1} x_{0}\right] \frac{1-z_{2} / z_{1}}{1+z_{2} / z_{1}}
$$

and

$$
|r|=\exp \left(-2 \phi_{\mathrm{R}}\right)=\left|\frac{1-z_{2} / z_{1}}{1+z_{2} / z_{1}}\right|
$$

In the considered case $U_{1}<E<U_{2}$ the value of $|r|$ is always equal to 1 exept one particular case $z_{1}=z_{2}$ when $r=0$. It is not a surprising result because if $z_{1}=z_{2}$, we have a constant potential in the whole region, which 


\section{O. I. HRYHORCHAK}

means that there is no reason for the wave to be reflected at point $x_{0}$. But the obtained results are valid also for the case of $U_{1}<U_{2}<E$ when both $z_{1}$ and $z_{2}$ are real. In this case $|r|$ is not equal to 1 .

On the basis of the obtained results, we can find expressions for both a transmission probability $T$ and a reflection probability $R$, namely

$$
R=\exp \left(-4 \phi_{\mathrm{R}}\right), \quad T=1-\exp \left(-4 \phi_{\mathrm{R}}\right)
$$

or

$$
R=\left|\frac{z_{2}-z_{1}}{z_{2}+z_{1}}\right|^{2}, \quad T=1-R .
$$

Now we can find the absolute value of a wave transmission amplitude coefficient

$$
|t|=\sqrt{1-|r|^{2}}
$$

But the question about the phase of $t$ is still relevant. Generally, if we want to find the phase of $t$ at the point where the wave is reflected, then we have a very simple relation, namely

$$
t=1+r
$$

But if one considers the potential energy of form (12) and is interested in the phase of $t$ at the point $x=b$, then the previous formula is not valid (it is valid only for the point $x=a$ if we consider the wave incidenting on the left). To find the correct formula, we have to use the following relations, which express the matching conditions for the wave function:

$C_{1} \exp \left[\frac{i m}{\hbar} \int^{a} Z_{1}\left(x^{\prime}\right) d x^{\prime}\right]=C_{2} \exp \left[\frac{i m}{\hbar} \int^{a} Z_{2}\left(x^{\prime}\right) d x^{\prime}\right]$

$C_{2} \exp \left[\frac{i m}{\hbar} \int^{b} Z_{2}\left(x^{\prime}\right) d x^{\prime}\right]=C_{3} \exp \left[i k_{3} b\right]$.

Remembering that $t=C_{3} /\left(C_{1} \exp \left[\phi_{1}\right]\right)$, we get

$$
t=\frac{\exp \left[\frac{i m}{\hbar} \int^{a} Z_{1}\left(x^{\prime}\right) d x^{\prime}\right] \exp \left[\frac{i m}{\hbar} \int^{b} Z_{2}\left(x^{\prime}\right) d x^{\prime}\right]}{\exp \left[\frac{i m}{\hbar} \int^{a} Z_{2}\left(x^{\prime}\right) d x^{\prime}\right] \exp \left[i k_{3} b+\phi_{1}\right]} .
$$

The multiplier $\exp \left[\phi_{1}\right]$ in the denominator of the expression for $t$ is due to $C_{1} \exp \left[\phi_{1}\right]$, which is the amplitude of the incidenting wave.

In the case of $r$ calculation for the potential energy of form (12), we have a formula very similar to (43), namely

$$
r=\frac{z_{1}-Z(a)}{z_{1}+Z(a)}
$$

when a wave incidents on the left and

$$
r=\frac{z_{2}-Z(b)}{z_{2}+Z(b)}
$$

when a wave incidents on the right.

\section{TUNELLING THROUGH A SINGLE RECTANGULAR BARRIER}

A solution of the scattering problem for a single barrier plays a significant role in the development of signalprocessing devices for different waves. We speak about single barrier resonant-tunneling structures which have high selective properties. In reality a tunnel barrier is implemented in the form of a semiconductor superlattice, a photonic or phononic crystal [26].

The simplest case of a single barrier system is a rectangular potential barrier:

$$
U(x)=\left\{\begin{array}{c}
U_{b}, \quad a \leq x \leq b \\
0, \quad(x<a) \wedge(x>b)
\end{array} .\right.
$$

The quantum wave impedance at the point $x=a$ can be calculated on the basis of the results of the previous section. Thus,

$$
Z(a)=z_{b} \frac{z_{0} \cosh \left(\gamma_{b} l\right)-z_{b} \sinh \left(\gamma_{b} l\right)}{z_{b} \cosh \left(\gamma_{b} l\right)-z_{0} \sinh \left(\gamma_{b} l\right)}
$$

where $l=b-a, i \gamma_{b}=(m / \hbar) z_{b}, z_{b}=\sqrt{2\left(E-U_{b}\right) / m}$, $z_{0}=\sqrt{E / m}$. Note that $\gamma_{b}$ is real ( $z_{b}$ is imaginary) when $E \leq U_{0}$ and $\gamma_{b}$ is imaginary ( $z_{b}$ is real) in the opposite case.

So now on the basis of formula (51) we easily get the following relation:

$$
\begin{aligned}
r & =\frac{\left(1-z_{b}^{2} / z_{0}^{2}\right) \sinh \left(\gamma_{b} l\right)}{2 z_{b} / z_{0} \cosh \left(\gamma_{b} l\right)-\left(1+z_{b}^{2} / z_{0}^{2}\right) \sinh \left(\gamma_{b} l\right)} \\
& =\frac{\left(1-\gamma_{b}^{2} / \gamma_{0}^{2}\right) \sinh \left(\gamma_{b} l\right)}{2 \gamma_{b} / \gamma_{0} \cosh \left(\gamma_{b} l\right)-\left(1+\gamma_{b}^{2} / z_{0}^{2}\right) \sinh \left(\gamma_{b} l\right)}
\end{aligned}
$$

To find $R$, we consider two cases separately: $E \leq U_{b}$ and $E>U_{b}$. In the first case $\left(E \leq U_{b}\right)$ we have that $\gamma_{b}$ and $z_{0}$ are real, $z_{b}$ is imaginary. So after introducing $k_{0}=$ $m z_{0} / \hbar\left(i k_{0}=\gamma_{0}, \gamma_{0}\right.$ is imaginary $)$ and $\varkappa_{b}=m z_{b} /(i \hbar)=$ $\sqrt{2 m\left(U_{b}-E\right)} / \hbar>0\left(z_{b}\right.$ is imaginary), we get

$$
R=|r|^{2}=\frac{\left(1+\varkappa_{b}^{2} / k_{0}^{2}\right)^{2} \sinh ^{2}\left(\varkappa_{b} l\right)}{4 \gamma_{b}^{2} / k_{0}^{2}+\left(1+\varkappa_{b}^{2} / k_{0}^{2}\right)^{2} \sinh ^{2}\left(\varkappa_{b} l\right)} .
$$

In the second case $\left(E>U_{0}\right)$ we have that $\gamma_{b}$ is imaginary, $z_{b}$ and $z_{0}$ are real. So after introducing $k_{b}=m z_{b} / \hbar\left(i k_{b}=\right.$ $\gamma_{b}$ ) we get

$$
R=|r|^{2}=\frac{\left(1-k_{b}^{2} / k_{0}^{2}\right)^{2} \sin ^{2}\left(k_{b} l\right)}{4 k_{b}^{2} / k_{0}^{2}+\left(1-k_{b}^{2} / k_{0}^{2}\right)^{2} \sin ^{2}\left(k_{b} l\right)} .
$$

To find $t$ we can use formula (50), in which for the simplification of calculations we assume that $a=0$ : 


$$
t=\frac{\exp \left[\gamma_{0} \int^{0} \tanh \left(\gamma_{0} x^{\prime}+\phi_{1}\right) d x^{\prime}\right] \exp \left[\gamma_{b} \int^{b} \tanh \left(\gamma_{b} x^{\prime}+\phi_{2}\right) d x^{\prime}\right]}{\exp \left[\gamma_{b} \int^{0} \tanh \left(\gamma_{b} x^{\prime}+\phi_{2}\right) d x^{\prime}\right] \exp \left[\gamma_{0} b+\phi_{1}\right]} .
$$

So we obtain

$$
t=\frac{\cosh \left(\phi_{1}\right) \cosh \left(\gamma_{b} b+\phi_{2}\right)}{\cosh \left(\gamma_{b} b+\phi_{2}\right) e^{\gamma_{0} b+\phi_{1}}}=\frac{1}{2}\left(1+e^{-2 \phi_{1}}\right) e^{-\gamma_{0} b}\left(\cosh \left[\gamma_{b} b\right]+\sinh \left[\gamma_{b} b\right] \tanh \left[\phi_{2}\right]\right)
$$

We get the values of $e^{-2 \phi_{1}}$ and $\tanh \left[\phi_{2}\right]$ on the basis of matching conditions for a quantum wave impedance:

$$
\begin{aligned}
& -z_{0} \tanh \left(\phi_{1}\right)=z_{b} \tanh \left(\phi_{2}\right) ; \\
& z_{b} \tanh \left(\gamma_{b} b+\phi_{2}\right)=z_{0} .
\end{aligned}
$$

Remembering that the second matching condition can be written as follows

$$
z_{b} \frac{\tanh \left(\gamma_{b} b\right)+\tanh \left(\phi_{2}\right)}{1+\tanh \left(\gamma_{b} b\right) \tanh \left(\phi_{2}\right)}=z_{0}
$$

we find that

$$
\begin{aligned}
& \tanh \phi_{2}=\frac{z_{0} / z_{b}-\tanh \left(\gamma_{b} b\right)}{1-z_{0} / z_{b} \tanh \left(\gamma_{b} b\right)}, \\
& 1+\exp \left[-2 \phi_{1}\right]=\frac{2 z_{0}}{z_{1} \tanh \left[\phi_{2}\right]+z_{0}} \\
& =\frac{\left(1-z_{0} / z_{b} \tanh \left(\gamma_{b} b\right)\right)}{1-\left(z_{b}^{2}+z_{0}^{2}\right) /\left(2 z_{0} z_{b}\right) \tanh \left(\gamma_{b} b\right)} .
\end{aligned}
$$

Substituting them into the initial relation and taking into account that $b=l$ (because of the $a=0$ assumption), we finally get

$$
\begin{aligned}
t & =\frac{2 z_{0} z_{b} \exp \left[-\gamma_{0} l\right]}{2 z_{0} z_{b} \cosh \left(\gamma_{b} l\right)-\left(z_{0}^{2}+z_{b}^{2}\right) \sinh \left(\gamma_{b} l\right)} \\
& =\frac{2 \gamma_{0} \gamma_{b} \exp \left[-\gamma_{0} l\right]}{2 \gamma_{0} \gamma_{b} \cosh \left(\gamma_{b} l\right)-\left(\gamma_{0}^{2}+\gamma_{b}^{2}\right) \sinh \left(\gamma_{b} l\right)}
\end{aligned}
$$

Note that $\gamma_{0}$ is imaginary regardless on the value of $U_{b}$.

When $E<U_{b}, z_{0}$ and $\varkappa_{b}$ are real and $z_{b}$ is imaginary. Thus, we have

$$
\begin{aligned}
T & =|t|^{2}=\frac{4 k_{0}^{2} \varkappa_{b}^{2}}{4 k_{0}^{2} \varkappa_{b}^{2} \cosh ^{2}\left(\varkappa_{b} l\right)+\left(k_{0}^{2}-\varkappa_{b}^{2}\right)^{2} \sinh ^{2}\left(\varkappa_{b} l\right)} \\
& \times\left\{1+\frac{1}{4}\left(\frac{k_{0}}{\varkappa_{b}}+\frac{\varkappa_{b}}{k_{0}}\right)^{2} \sinh ^{2}\left(\varkappa_{b} l\right)\right\}^{-1}
\end{aligned}
$$

For $E>U_{0}, z_{0}$ and $z_{b}$ are real and $\gamma_{b}$ is imaginary, $i k_{b}=\gamma_{b}$ and

$$
\begin{aligned}
T & =|t|^{2}=\frac{4 k_{0}^{2} k_{b}^{2}}{4 k_{0}^{2} k_{b}^{2} \cos ^{2}\left(k_{b} l\right)+\left(k_{0}^{2}+k_{b}^{2}\right)^{2} \sin ^{2}\left(k_{b} l\right)} \\
& \times\left\{1+\frac{1}{4}\left(\frac{k_{0}}{k_{b}}-\frac{k_{b}}{k_{0}}\right)^{2} \sin ^{2}\left(k_{b} l\right)\right\}^{-1} .
\end{aligned}
$$

\section{CONCLUSIONS}

Very often the most important and interesting aspect (especially in the area of practical application) of wave propagation is the issue of energy transmission. This includes the calculation of such parameters as transmission and reflection coefficients and finding conditions for standing waves to form and resonant states to appear. In this case, the impedance concept is very fruitful because it gives the possibility to calculate the mentioned parameters in an easier way than by using other methods.

This is clearly seen in the example of calculation of the reflection coefficient for a singular rectangular barrier. We got the result for $r$ (55) very easily by using the quantum wave impedance approach. Other approaches, namely, the classical method based on the direct solving of the Schrödinger equation and the transfer matrix technique require much more effort. In this case, the classical method implies constructing and solving two determinants of $4 \times 4$ size, while within the transfer matrix method we have to build three matrices of $2 \times 2$ and then multiply them in a correct order.

What is the reason for this advantage? As we mentioned earlier, the main reason is that the solution of the first order differential equation (for the quantum wave impedance function) contains only one undefined constant, while the second order (Schrödinger) equation has two undefined constants. Thus, a consecutive calculation of quantum wave impedance is much easier compared to a wave function calculation, because instead of two matching conditions it is enough to use only one.

All this means that the quantum wave impedance approach is a very efficient tool for the calculation of the probability amplitudes of the particle reflection and 


\section{O. I. HRYHORCHAK}

transition, especially in the case of systems with many wells and barriers, as well as for finding the conditions of resonant tunneling and bound states.

Concluding, we have to say that in general the appli- cation of impedance models for quantum-mechanical systems allows simplifying and generalizing the process of studying these systems significantly. This clearly can be seen from articles [27-30].
[1] O. Heaviside, Electrician 17, 212 (1886); reprinted as Electrical Papers, Vol. II (Macmillan \& Co., LondonNew York, 1892), p. 64.

[2] A. E. Kennelly, Trans. Am. Inst. Electr. Eng. 10, 172 (1893); https : //doi .org/10.1109/T-AIEE.1893.47 68008.

[3] A. Šabanović, K. Ohnishi, Motion Control Systems (John Wiley and Sons (Asia) Pte Ltd, 2011); https://doi.or $\mathrm{g} / 10.1002 / 9780470825754$.

[4] L. E. Kinsler, A. R. Frey, A. B. Coppens, J. V. Sanders, Fundamentals of Acoustics, 4th ed. (John Wiley and Sons Ltd, 2000).

[5] A. Guran, R. Mittra, P. J. Moser, Electromagnetic Wave Interactions (World Scientific, 1996); https://doi.org/ 10.1142/3071.

[6] A. N. Khondker, M. R. Khan, A. F. M. Anwar, J. Appl. Phys. 63, 5191 (1988); https://doi.org/10.1063/1.34 1154.

[7] A. F. M. Anwar, A. N. Khondker, M. R. Khan, J. Appl. Phys. 65, 2761 (1989); https://doi.org/10.1063/1.34 2766.

[8] A. N. Khondker, J. Appl. Phys. 67, 6432 (1990); https: //doi.org/10.1063/1.345116.

[9] A. Haque, M. Haque, M. R. Khan, J. Appl. Phys. 68, 1661 (1990); https://doi.org/10.1063/1.346648.

[10] R. A. Morrisey, M. A. Alamb, A. N. Khondker, Physica B 182, 61 (1992); https://doi.org/10.1016/0921-452 6(92) 90572-A.

[11] A. N. Khondker, M. A. Alam, Phys. Rev. B 44, 5444 (1991); https://doi.org/10.1103/PhysRevB. 44.5444.

[12] A. N. Khondker, M. A. Alam, Phys. Rev. B 45, 8516 (1992); https://doi.org/10.1103/PhysRevB.45.8516.

[13] R. Kaji, K. Hayata, M. Koshiba, Electron Commun Jpn 75, 44 (1992); https://doi.org/10.1002/ecjb.44207 50905.

[14] E. A. Nelin, Tech. Phys. 49, 1464 (2004); https://doi. org/10.1134/1.1826191.

[15] E. A. Nelin, Tech. Phys. 50, 1511 (2005); https://doi. org/10.1134/1.2131963.
[16] E. A. Nelin, Tech. Phys. 51, 1101 (2006); https://doi. org/10.1134/S1063784206080238.

[17] E. A. Nelin, Tech. Phys. 52, 1222 (2007); https://doi. org/10.1134/S1063784207090198.

[18] E. A. Nelin, Tech. Phys. 54, 953 (2009); https://doi. org/10.1134/S1063784209070044.

[19] D. V. Khatyan, M. A. Gindikina, E. A. Nelin, Visn. NTUU KPI Ser. Radiotekh. Radioaparatobud. 62, 100 (2015); https://ela.kpi.ua/handle/123456789/15 330.

[20] A. I. Nazarko, U. F. Tymofeeva, V. I. Nelin, N. A. Popsuy, Tekhn. Konstr. Elektr. Appar. 6, 38 (2009); ht tp://dspace.nbuv.gov.ua/handle/123456789/52329.

[21] A. Ashby, MSc thesis (Faculty of the Graduate School of the University of Colorado, 2016).

[22] E. A. Nelin, Ya. L. Zinher, V. I. Popsui, Visn. NTUU KPI Ser. Radiotekh. Radioaparatobud. 71, 46 (2017); https://doi.org/10.20535/RADAP. 2017.71.46-51.

[23] E. A. Nelin, A. V. Shulha, Ya. L. Zinher, Visn. NTUU KPI Ser. Radiotekh. Radioaparatobud. 73, 63 (2018); https://doi.org/10.20535/RADAP . 2018.73.63-68.

[24] D. R. Hartree, Math. Proc. Camb. Philos. Soc. 24, 89 (1928); https://doi.org/10.1017/S0305004100011 919.

[25] Y. Nogami, F. M. Toyama, Phys. Rev. A 5793 (1998); https://doi.org/10.1103/PhysRevA.57.93.

[26] E. A. Nelin, A. V. Sergiyenko, Visn. NTUU KPI Ser. Radiotekh. Radioaparatobud. 36, 96 (2008); http://ra dap.kpi.ua/radiotechnique/article/view/729/.

[27] E. A. Nelin, Phys.-Uspekhi 50, 293 (2007); https://do i.org/10.1070/pu2007v050n03abeh006091.

[28] V. O. Bojko, B. M. Berezjanskyi, E. A. Nelin, Visn. NTUU KPI Ser. Radiotekh. Radioaparatobud. 34, 106 (2007).

[29] E. A. Nelin, M. V. Vodolazka, Visn. NTUU KPI Ser. Radiotekh. Radioaparatobud. 56, 129 (2014); http:// radap.kpi.ua/radiotechnique/article/view/752.

[30] R. S. Akhmedov, E. A. Nelin, Visn. NTUU KPI Ser. Radiotekh. Radioaparatobud. 34, 102 (2007). 


\title{
ПЕРЕФОРМУЛЮВАННЯ ЗАДАЧІ ВІДБИВАННЯ ТА ПРОХОДЖЕННЯ В ТЕРМІНАХ ФУНКЦІЇ КВАНТОВО-МЕХАНІЧНОГО ІМПЕДАНСУ
}

\author{
O. I. Григорчак \\ Кафедра теоретичної фізики імені професора Івана Вакарчука \\ Лъвівсъкого націоналъного університету імені Івана Франка, \\ вул. Драгоманова 12, Лъвів, Украӥна \\ Orest.Hryhorchak@lnu.edu.ua
}

На основі одновимірного рівняння Шредингера, переписаного з використанням логаритмічної похідної від хвильової функції, було отримане нелінійне диференціальне рівняння першого порядку (типу Ріккаті) для функції квантово-механічного імпедансу. Множник, що пов'язує функцію квантово-механічного імпедансу з логаритмічною похідною від хвильової функції, забезпечує простий і фізично змістовний вигляд виразу для густини потоку ймовірності, а саме: як добуток квадрата модуля хвильової функції на дійсну частину функції квантово-механічного імпедансу.

Розглянуто як задачу розсіяння, так і задачу пошуку зв'язаних станів. Обидві переформульовано в термінах квантово-механічного імпедансу, а також знайдено умови для пошуку власних рівнів енергії та отримано формули для амплітуд проходження й відбивання квантової частинки від потенціального бар'єра довільної форми.

Нелінійне диференціальне рівняння для функції квантово-механічного імпедансу було розв'язане для константного потенціалу. Продемонстровано, як використати отримані результати в задачі розсіяння на прямокутному потенціальному бар'єрі. Знайдені вирази для амплітуд та коефіцієнтів проходження й відбивання збігаються з уже відомими результатами.

Обговорено переваги підходу квантово-механічного імпедансу порівняно з класичним, зокрема в контексті застосування чисельних методів для вивчення поведінки квантової частинки в потенціалах зі складною просторовою структурою.

Ключові слова: рівняння Шредингера, квантово-механічний хвильовий імпеданс, розсіяння, тунелювання. 